\title{
Investigation of Molecular Functions of Zinc Finger Protein (ZNF71) in Non- Small Cell Lung Cancer
}

\author{
Rehab O. Mohamed \\ romohamed@hsc.wvu.edu
}

Follow this and additional works at: https://researchrepository.wvu.edu/etd

\section{Recommended Citation}

Mohamed, Rehab O., "Investigation of Molecular Functions of Zinc Finger Protein (ZNF71) in Non- Small Cell Lung Cancer" (2020). Graduate Theses, Dissertations, and Problem Reports. 7750.

https://researchrepository.wvu.edu/etd/7750

This Thesis is protected by copyright and/or related rights. It has been brought to you by the The Research Repository @ WVU with permission from the rights-holder(s). You are free to use this Thesis in any way that is permitted by the copyright and related rights legislation that applies to your use. For other uses you must obtain permission from the rights-holder(s) directly, unless additional rights are indicated by a Creative Commons license in the record and/ or on the work itself. This Thesis has been accepted for inclusion in WVU Graduate Theses, Dissertations, and Problem Reports collection by an authorized administrator of The Research Repository @ WVU. For more information, please contact researchrepository@mail.wvu.edu. 
Graduate Theses, Dissertations, and Problem Reports

2020

Investigation of Molecular Functions of Zinc Finger Protein

(ZNF71) in Non- Small Cell Lung Cancer

Rehab O. Mohamed 
Investigation of Molecular Functions of Zinc Finger Protein (ZNF71) in NonSmall Cell Lung Cancer

\author{
Rehab Mohamed \\ A thesis submitted to the School of Medicine \\ at West Virginia University \\ in partial fulfillment of the requirements for the degree of \\ Master's degree \\ in \\ Biomedical Science
}

\author{
Lan Guo, PhD, Chair \\ Timothy Eubank, PhD \\ Alexey Ivanov, PhD
}

Department of Biomedical Sciences

Morgantown, WV

2020

Keywords: ZNF71, NSCLC, KRAB-ZNF, KRAB less, ZNF71, EMT,

(C) 2020 Rehab Mohamed 


\title{
Abstract \\ Investigation of Molecular Functions of Zinc Finger Protein (ZNF71) in Non- Small Cell Lung Cancer Rehab Mohamed
}

\begin{abstract}
Non-small cell lung cancer (NSCLC) is the first cause of tumor-related death worldwide. Despite the advancement in surgical and medical treatment, the prognosis of the disease remains poor $^{1}$. To date, there is no available molecular assay to predict the benefit of therapy or tumor recurrence ${ }^{2}$. ZNF71 was reported in our previous study, which identified a 7-gene predictive signature for the clinical benefit of chemotherapy and prognosis in NSCLC ${ }^{2}$. The study concluded that patients who have higher ZNF71 protein expression have better survival. For this reason, we decided to study the molecular function of ZNF71 and how it may affect apoptosis, proliferation, and/or epithelial-mesenchymal transformation (EMT) in NSCLC. The study is restricted by the regulations of COVID-19 social distancing, so many experiments were stopped and recommended for future work. We used a Taxol-resistant cell line H460-R derived from the Taxol-sensitive H460-P NSCLC cell line to compare the level of ZNF71. H460-P cells express a higher level of ZNF71 mRNA, and Taxol induces it slightly. Also, we found that the ZNF71 mRNA level is higher among epithelial NSCLC, and lower in mesenchymal-like-cells. Furthermore, KRAB containing ZNF71 is expressed dominantly in mesenchymal NSCLC, while KRAB-less ZNF71 is expressed in both mesenchymal and epithelial and its level is always higher than KRAB isoform except for in H460-R cells. This suggests that a KRAB to KRAB-less ratio could has a role in the cells' fate and the patients' prognosis, but further studies are required and recommended.
\end{abstract}




\section{DEDICATION}

To the spirit of the women who raised me, my maternal grandmother, who has been a rock of stability throughout my life;

My parents, whose loving spirit sustains me still;

And to my husband, for unlimited support in my life; And to my kids who provide me the feeling of happiness of motherhood. 


\section{ACKNOWLEDGMENTS}

First and foremost, I would like to especially thank my mentor and committee chairperson Dr. Lan Guo for all the support and guidance she provided for my academic, research and professional development. Also, for her caring guidance and help in writing my thesis.

I appreciate the guidance and comments which I have received from my supervising committee members, Dr. Tim Eubank and Dr. Alexey Ivanov. For their invaluable support, and valuable advice and suggestions.

Special thanks for Dr. Clay Marsh, for his valuable suggestions and advice.

I would like to express my sincere gratitude to Dr. Marieta Gencheva, a scientist in the Eubank laboratory, Health Science Center in WVU, for her invaluable support, guidance, patience and valuable suggestions throughout my master's work

I would like to say thanks to my friend and lab mate, Qing Ye. As well as to the whole Eubank lab members, Dr. Elena Pugacheva and her lab members, Dr. Siva Kolluri and Dr. Ivan Martinez and his lab members for providing us with some cell lines during our research.

Last, but far from least, I would like to express my deep appreciation, gratitude, and love for my husband who supported me throughout this journey. Without him, I could have never made it to where I am today. 


\section{Contents}

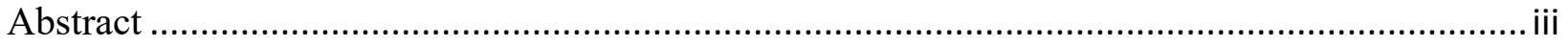

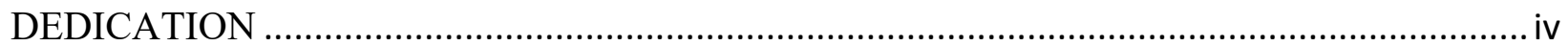

ACKNOWLEDGMENTS ...................................................................................................

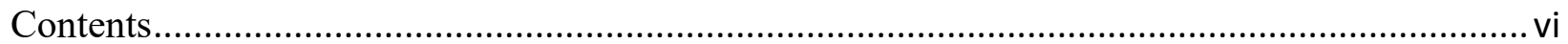

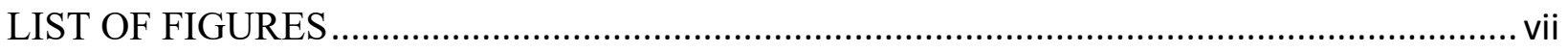

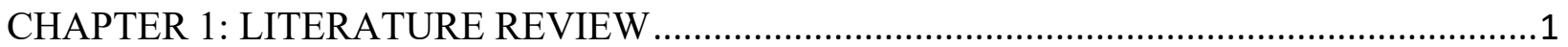

1.1 Zinc Finger Proteins and tumors ................................................................................... 3

1.2 Zinc finger protein 71(ZNF71) ................................................................................. 4

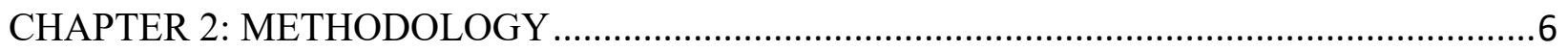

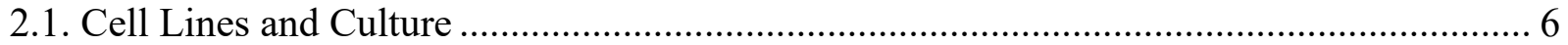

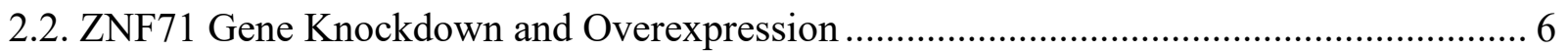

2.3 Quantitative Real Time Polymerase Chain Reaction qRT-PCR …………………............. 7

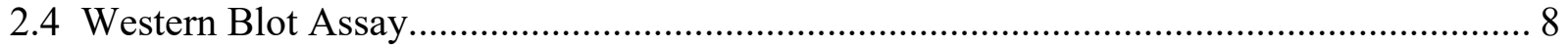

2.5 Annexin V and Propidium Iodide Assay …………....................................................... 8

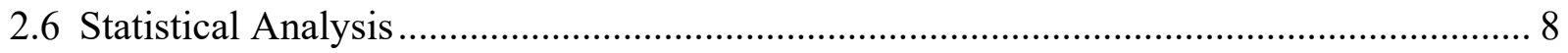

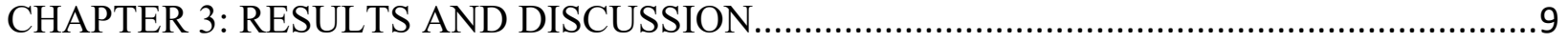

3.1 H460-R Cells are Highly Resistant to Taxol Treatment ................................................... 9

3.2. Validation of the ZNF71 Antibodies by Overexpression of the Protein ............................. 9

3.3. ZNF71 mRNA Levels are Higher in H460-P than in H460-R......................................... 10

3.4 H460-R Cells Response to Taxol Treatment Slightly Affected by Changing the Levels of

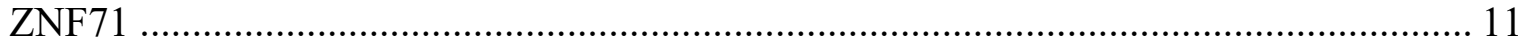

3.5. ZNF71 is Expressed in Different Amounts in Different non-Small Cell Lung Cancer Cells

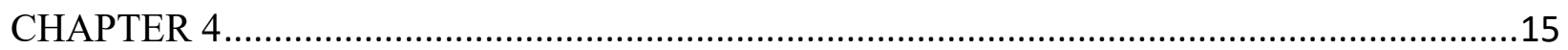

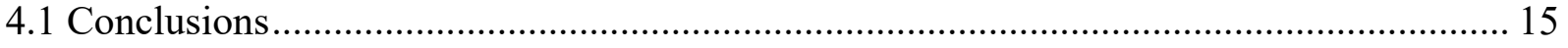

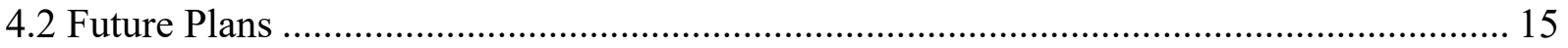

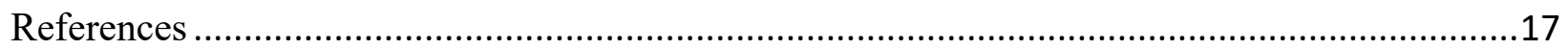




\section{LIST OF FIGURES}

Figure 1. 1 Schematic Figure Represents The Structure Of C2H2, RING, PHD, And LIM Zinc

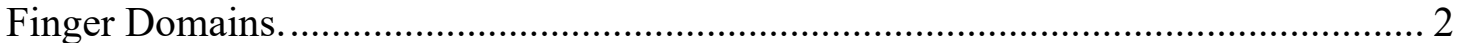

Figure 1. 2 KRAB-ZNF structure, KRAB-A and KRAB-B located at N-terminus. DNA binding motifs .................................................................................................. 3

Figure 1. 3. Schematic figure explain the structure of KRAB and KRAB-less ZNF71 isoforms .. 4

Figure 3. 1. H460-R cells are Taxol-resistant. H460-P (A) and H460-R (B) cells were treated either with DMSO or Taxol with the indicated doses..................................................... 9

Figure 3. 2: ZNF71 antibodies validation.......................................................................... 10

Figure 3. 3: Comparison of the ZNF71 mRNA level in resistance cells H460-R and parental cells

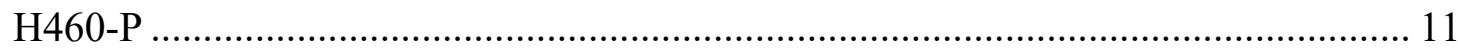

Figure 3. 4 Effect of ZNF71 gene knockdown on H460-R cells response to Taxol................... 12

Figure 3.5 .A. ZNF71 level is different in epithelial and mesenchymal cell lines. .................. 13

Figure 3. 6 Relative expression ZNF71 mRNA isoform among different NSCLC cell lines. ..... 14 


\section{CHAPTER 1: LITERATURE REVIEW}

Zinc finger proteins (ZNF) are the largest family of proteins not only in human cells but also in most eukaryotes, representing about $3 \%$ of the total human genome ${ }^{1}$. They were discovered for the first time in 1982 by Miller and Klug while studying the transcription factor IIIa (TF IIIa) in Xenopus Laevis ${ }^{1,2}$. The authors observed and discovered a novel repeating motif within the protein, which was later named zinc finger ${ }^{1}$ - composed of histidine and cysteine amino acids coordinated by zinc ions. This motif has the ability to bind to DNA, RNA, and other cellular proteins $^{3}$. Therefore, ZNFs have a wide range of functions according to their molecular structure and binding site. ZNFs can be involved in DNA repair, degradation of proteins, signal transductions, migration of cells, regulation of apoptosis, lipid binding, and transcription regulation ${ }^{3,2}$. There are many subtypes of ZNFs classified according to different combinations of cysteine and histidine coordinated by zinc ion. The most common one is named $\mathrm{C} 2 \mathrm{H} 2 \mathrm{ZNF}$, and is composed of two histidines in one chain and two cysteines in another chain coordinated by zinc ion, in the form of C-x-C-x-H-x-H. The domain functions by binding to specific DNA sequences. The primary contact made by the alpha helix that binds to the major groove of the DNA through primary hydrogen bond interaction from positions 1,3 and 6 to three successive bases, figure 1.2, and through secondary interaction from position 2 . There is a highly conserved linker region TGEKP in almost half of $\mathrm{C} 2 \mathrm{H} 2$ zinc finger protein that are believed to play a role in the affinity of ZNF to bind DNA2. Other less common zinc finger domain types include RING, PHD, and LIM, figure 1.1. Really Interesting New Gene (RING) are a group of proteins which include the RING-motif C-x-C-x-C-x-H-x-C-x-C-x-C-x-C. E3 ubiquitin ligases are among the proteins belonging to this group, one of which is the mouse double minute2 MDM2 gene, believed to be involved in cancer progression ${ }^{3}$. The Plant Homeodomain PHD is a motif which has the ability to remodel the chromatin through epigenetic modification. Lin-11, Isl-11 and Mec-3 LIM class play an important role in actin and cytoskeletal interaction as well as focal adhesion ${ }^{3}$. 


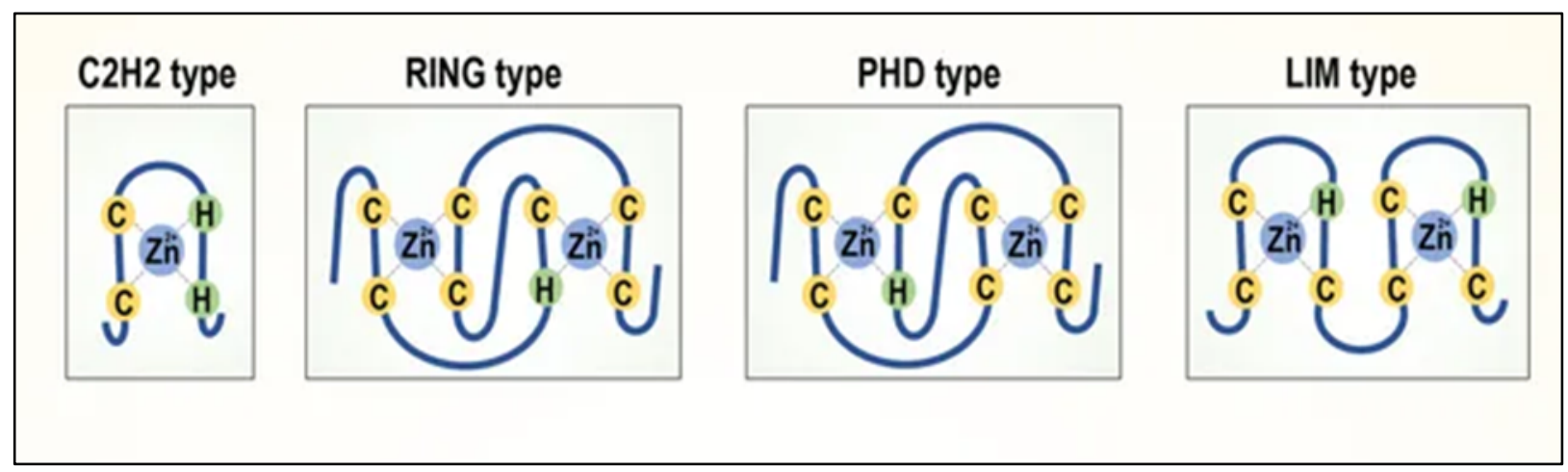

Figure 1. 1 Schematic Figure Represents The Structure Of C2H2, RING, PHD, And LIM Zinc Finger Domains.

The most exciting subgroup of the ZNF proteins are the Krupple-associated box domain zinc finger protein (KRAB-ZNF).

KRAB-ZNF are a family of transcriptional repressors with a diverse array of functions, most notably the silencing of transposable elements ${ }^{4}$. They contain an N-terminal KRAB domain and a C-terminal $\mathrm{C} 2 \mathrm{H} 2$ zinc finger group. The KRAB domain is composed of around 75 amino acids $^{5}$, and there are two types of KRAB domains; KRAB box A, which is known to be responsible for the repressive activity of the zinc finger, and box $\mathrm{B}$, the function of which is to aid the action of box A. Both boxes are located at the N-terminus of the zinc finger 1.2. The repressor action of KRAB-ZNF requires the recruitment of KRAB Associated Protein 1, also know Tripartite Motif Protein 28, KAP1/TRIM28. 


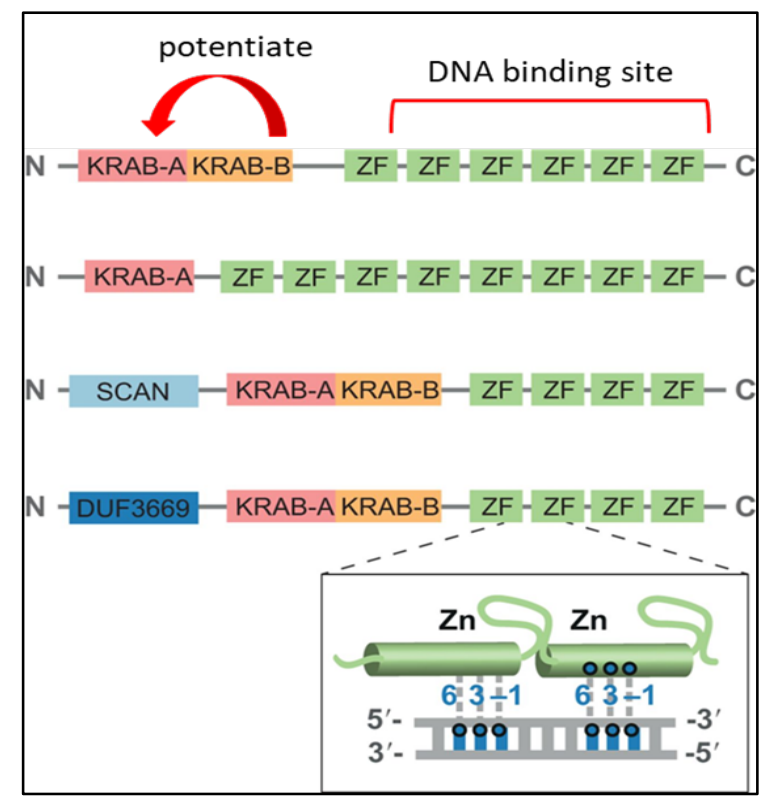

Figure 1. 2 KRAB-ZNF structure, KRAB-A and KRAB-B located at $N$-terminus. DNA binding motifs located at C-terminus ${ }^{6}, Z N F$ s binds DNA through primary hydrogen-bond interactions from helical positions 1,3, and 6.

KAP1 functions as a scaffold complex composed of histone methyl transferase (SETDB1), Heterochromatin Protein-1 (HP-1), Nucleosome Remodeling and Deacetylation (NuRD), and DNA Methyl Transferase ${ }^{6}$. When KRAB-ZNF recruits KAP-1, the repressor complex formed leads to heterochromatin formation. On the other hand, ZNFs bind to DNA mainly through their C- terminus domain which comprises primarily of tandem repeats with the motif $\left(\mathrm{CX}_{2-4} \mathrm{CX}_{12}\right.$ HX 2-6 H), this activity starts as early as the first few days of embryonic life, results in transgenerational imprinting.

\subsection{Zinc Finger Proteins and tumors}

Since the KRAB domain binds directly to KAP1, most of the suppressive functions of KRAB-ZNF is mediated through KAP1. The role of KAP1 in cancer has just emerged in the field. Using microarray analysis, KAP1 expression level was found to be increased with the increase of the clinical phase of breast tumors ${ }^{7}$. Also, overexpression of many KRAB-ZNFs and KAP1 in breast tumors was reported, while knockdown of KAP1 led to a suppression of tumor cells proliferation, growth, and metastasis. Furthermore depletion of KAP1 led to a decrease in the expression KRAB-ZNF proteins ${ }^{8}$. There are many other studies ongoing to find out the effect of zinc finger proteins on the prognosis of non-small cell lung cancer patients. RB-associated KRAB 
zinc finger (BRAK) interacts with the tumor suppressor retinoblastoma gene, RB. The RBAK is upregulated in non-small cell lung cancer (NSCLC) and has a potential effect on tumor cells migration and invasion. Furthermore, higher expression of RBAK was found to be associated with short survival in cancer patients (NSCLC) ${ }^{8}$. On the other hand, zinc finger protein 668 (ZNF668) protein expression is down regulated in tumor tissue in comparison to surrounding normal lung tissue. It also was found to be down regulated in lymph nodes metastasis phase, and restoration of ZNF668 expression led to down regulation of Snail and increase E-cadherin protein level ${ }^{9}$.

\subsection{Zinc finger protein 71(ZNF71)}

ZNF71 is a member of the Krupple C2H2-types zinc finger protein family. It has two isoforms derived by alternative splicing, a 489 AA short isoform and a 549 AA long isoform. The two isoforms differ by the inclusion of an alternative exon, which results in inclusion of a KRAB domain in the long isoform. Both ZNF71 isoforms contain identical C-termini comprising 13 $\mathrm{C} 2 \mathrm{H} 2$ type zinc finger, as shown in figure 1.3.

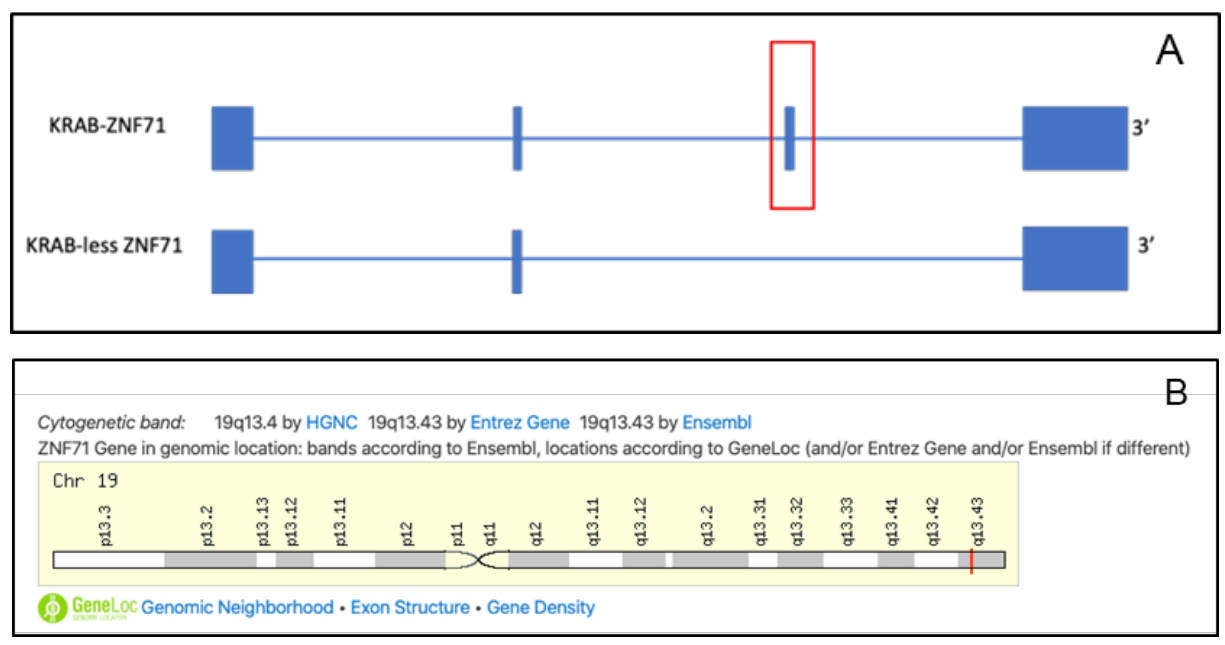

Figure 1. 3. A, schematic figure explaining the structure of KRAB and KRAB-less ZNF71 isoforms. KARB domain was labeled with a red box. B, ZNF71 gene location in long arm of chromosome 19.

ZNF71 was first identified by Mataki et al. as a zinc finger protein induced by TNF alpha in human umbilical vein endothelial cells ${ }^{10}$. It was named EZFIT, Endothelial Zinc finger protein Induced by TNF alpha. In this study, ZNF71 was shown to be expressed at a high level in placenta, with expression also detected in brain, testis, pancreas, heart, small intestine, muscle, uterus, 
prostate and peripheral blood leukocytes. Tissues which did not express ZNF71 were liver, lung, colon, stomach, the salivary gland, and the thyroid gland. ZNF71 was also reported in our previous study which identified a 7-gene predictive signature for clinical benefit of chemotherapy and prognosis in NSCLC ${ }^{11}$. Prior to our study, there were no available predictive molecular assays to speculate the benefits of chemotherapy or the risk of tumor recurrence in NSCLC patients. Therefore, our study aimed to develop a multi-gene assay to predict the clinical benefits of the chemotherapy for patients who suffer from NSCLC. A total of 160 genes were identified from a microarray analysis, and the mRNA expression of these genes were analyzed using qRT-PCR. Using independent validation patient cohorts, a 7-gene signature was identified from the training cohort. Within this model, patients were stratified into two prognostic groups with distinct postsurgical disease-specific survival and recurrence-free survival. To validate the functional involvement of the identified 7-gene signature, immunohistochemistry staining (IHC) was used to evaluate the protein expression of these biomarkers. The antibodies to biomarkers with good quality in formalin fixed paraffin embedded (FFPE) NSCLC tissues were further quantified with Automated Quantitative Analysis (AQUA) on tissue microarrays (TMA) constructed at Yale Pathology Laboratory. For ZNF71 protein, an AQUA score cutoff point was identified to define a patient's prognostic stratification. It was concluded that patients with higher protein expression have better survival, and the protein expression of ZNF71 was concordant with its mRNA expression in independent NSCLC patient cohorts that the higher mRNA expression of ZNF71 was associated with better patient survival and chemo-sensitivity to traditional therapy of NSCLC $(\text { Taxol }+ \text { platinum based therapy })^{11}$. Furthermore, there was a $7 \%$ loss of DNA copy number in NSCLC tissue in comparison to normal lung tissue ${ }^{11}$. All of the evidence suggest the importance of ZNF71 as a prognostic marker, or even a novel therapeutic target in the future. For this reason, we decided to study the molecular functions of the ZNF71 gene, and how it may affect proliferation, apoptosis, EMT, and/or chemotherapy response in NSCLC cells. 


\section{CHAPTER 2: METHODOLOGY}

\subsection{Cell Lines and Culture}

Primary cell lines and non-small cell lung cancer (NSCLC) cells used in this project include; Human Small Airway Epithelial Cells (SAEC) were maintained in Small Airway Epithelial Cell Growth Medium Bulletkit ${ }^{\mathrm{TM}} \mathrm{SAGM}^{\mathrm{TM}}$ medium (Lonza, catalog number CC-3118). Human Bronchial/ Tracheal Epithelial Cells with Retinoic Acid (NHBE) cells were maintained in Bronchial Epithelial Cell Growth Medium BulletKit ${ }^{\mathrm{TM}}$ BEGMTM medium (Lonza, catalog number CC-3170), H358, H441, H820, H1395, H23, H513, H1299, and A549 NSCLC cells were all maintained in Dulbecco's Modified Eagle's Medium (DMEM), supplemented with 10\% of fetal bovine serum and 1\% penicillin/streptomycin. H460 large cell lung cancer cells (H460-P) and its derivative $\mathrm{H} 460-\mathrm{R}^{12}$, which are resistant to Taxol treatment, were kindly provided by Dr.Siva Kumar Kolluri, the Siva Kolluri laboratory at the Oregon State University as part of their collaboration with the Guo laboratory. These cell groups were maintained in Roswell Park Memorial Institute (RPMI-1640) medium, supplemented with $10 \%$ fetal bovine serum and $1 \%$ penicillin/streptomycin. All primary and non-small cell lung cancer cells were incubated at $37 \mathrm{C}^{\circ}$ and $5 \% \mathrm{CO}_{2}$ concentration.

\subsection{ZNF71 Gene Knockdown and Overexpression}

For knockdown purposes, we used a pool of three target-specific 19-25 nucleotide length ZNF71 siRNAs, purchased from Santa Cruz Biotechnology (catalog number SC-77001). As scrambled controls, we used control siRNA-A (catalog number SC-37007) and control siRNA-D (catalog number SC-44232). H460 cells were plated in six-well plates at 250,000 cells per well and were transfected after $24-48$ hours, when cells confluence reached $\sim 60-70 \%$. Transfection was performed with Lipofectamine RNAiMAX transfection reagent (Thermo Fisher Scientific) in Opti-MEMTM reduced serum medium (Thermo Fisher Scientific), according to manufacturer's protocol RNA was isolated 72 hours post-transfection.

For overexpression purposes, the ZNF71 Plasmid construct was purchased from the PlasmID database (https://plasmid.med.harvard.edu/PLASMID/OrderOverview.jsp) maintained by Harvard University (catalog number HsCD00412101), ZNF175 plasmid construct used as a control (catalog number HsCD00421069), and pLU-GFP vector was also used as a control. For 
HEK-293T cell transfection, calcium phosphate was used as a transfection reagent, which is commonly used to introduce DNA into eukaryotic cells to obtain both transient and permanent transfections. The approach is based on mixing HEPES-buffered saline containing $\mathrm{Na}_{3} \mathrm{PO}_{4}$ with $\mathrm{CaCl}_{2}$ containing the DNA. The DNA calcium phosphate stick to the cell membrane then is taken by cellular endocytosis. For the NSCLC transfection reagent, Lipofectamine was purchased from Thermo Fisher Scientific (catalog number L3000008). Cells were cultured in six-well plates seeding 400,000-500,000 cells per plate according to the transfection type; reverse, serum-free medium, or regular transfection. $15 \mu \mathrm{g}$ of plasmid in $50 \mu \mathrm{CaCl}$ was used to transfect $\mathrm{HEK}-293$ in a $35 \times 10 \mathrm{~mm}$ cell culture dish.

\subsection{Quantitative Real Time Polymerase Chain Reaction qRT-PCR.}

Total RNA was extracted from various cell lines using the RNeasy Mini kit (Qiagen). According to manufactures' protocol, RNA was eluted in $30 \mu \mathrm{l}$ deionized water and kept at $-80^{\circ} \mathrm{C}$ until use. Concentration of RNA was determined with NanoDrop, and RNA purity was verified by determining the A260/A230 ratio. One microgram of total RNA was reverse transcribed using SuperScriptIII First Strand Synthesis SuperMix for qRT-PCR (Invitrogen). For quantitative real time PCR, either SYPR Select Master Mix (Thermo Fisher) or TaqMan master mix (IDT) was used, depending on the primers/probes used. All primers were purchased from IDT. There are three sets of ZNF71 primers:

Set1 (will amplify both isoforms of the gene) primer F: 5'- CAGCACTTCAGACCTCAGTAAG3', primer R: 5'-TTGGTGCTTTATCAGGGACG-3', Set 2, KRAB-Less ZNF71, primer F: 5'GCCTGTCTTCCTATTCACCG-3', primer R: 5'-CATTTCAGGTCTAGTCTCCCAG-3', probe: /56-FAM/AGC CAT CCC /ZEN/TCT GCT GCC C/3IABkFQ/

Set3, KRAB-ZNF71, primer F: 5'- GACGTTCAGGGATGTGACTG-3', primer R: 5'TTCAGGTCTAGTCTCCCAGTC-3', probe: /56-FAM/AGG TCC TTC /ZEN/TGG GCA GGC $\mathrm{TC} / 3 \mathrm{IABkFQ} /$.

The PCR reactions were loaded in a 384-well plate in triplicate. The expression level of ZNF71 was calculated using the Comparative Threshold $\left(2^{\wedge}\right.$-ddCt $)$ method and normalized to UBC or RPL4 housekeeping genes. UBC primer set, primer F: 5'-GATTTGGGTCGCAGTTCTTC-3', primer R: 5'-CCTTATCTTGGATCTTTGCCTTG-3'. RPL4 (Cat. N PPH13915A SABiosciences) ${ }^{13}$. For SYBR Green, PCR melting curve analysis was performed to verify the reactions had a single product. 


\subsection{Western Blot Assay}

Cells were lysed in Gel Lysis Buffer (GLB), GLB composed of 50 mM Tris-HCl, pH 6.8, $2 \%$ SDS, $10 \%$ Glycerol. Total protein was quantified using the BCA assay (Pierce). An equal amount of protein lysate $(20-30 \mu \mathrm{g})$ was loaded and separated on 4-12\% Bis-Tris gels, then transferred to polyvinylidene difluoride (PVDF) membrane (Fisher). ZNF71 antibodies were purchased from Sigma Aldrich (Cat No. AV39497), and GeneTex (Cat No. GTX116553). Standard chemiluminescence was used to detect the protein bands.

\subsection{Annexin V and Propidium Iodide Assay}

Cells were seeded in six-well plates at 250,000-500,000 cells per well, then treated with 1, 5 , 10, or $50 \mathrm{nM}$ Taxol either 24 hours post-plating or 72 hours post ZNF71 siRNA transfection. DMSO was used as a vehicle control. An Annexin V binding assay was performed using the FITC Annexin V/Dead Cell Apoptosis Kit with FITC Annexin V and PI for flow cytometry (Invitrogen). PI is the standard reagent to exclude non-viable cells from flow cytometry assay, i.e., to calculate cell death. Flow cytometry was performed at the Flow Cytometry and Single Cell Core Facility at WVU on the LSRFortessa machine.

\subsection{Statistical Analysis}

A mixed effect model was used to assess the difference between two conditions, using $\mathrm{R}$ package Ime4. Linear mixed-effect model were used to assess the difference between different conditions as fixed effects (biological samples as random effects), taking into account the correlation between technical replicate. The p-value was calculated based on asymptotic Zdistribution. 


\section{CHAPTER 3: RESULTS AND DISCUSSION}

\subsection{H460-R Cells are Highly Resistant to Taxol Treatment}

Since we were interested to study the role ZNF71 plays in chemotherapy resistance in lung cancer, we wished to verify that we have a cell culture model of chemo-resistance. We employed H460-P cells, which are sensitive to Taxol, and a derivative cell line, H460-R, reported to be Taxol resistant. Both H460-R and H460-P were plated in 6-well plates in three biological replicates and incubated with either $10 \mathrm{nM}$ or $50 \mathrm{nM}$ Taxol for 48 hours, with DMSO used as vehicle control. Supernatants were centrifuged to collect floating cells which were combined with trypsinized cells and subsequently stained with Annexin V and PI. Percentage of apoptotic/dead cells was determined by flow cytometry. As shown in figure 3.1(A), after 48 hours DMSO treatment, the percent of live H460-P cells is $88.5 \%$, but drops after treatment with $10 \mathrm{nM}$ Taxol to $34 \%$ and as low as $27 \%$ when the cells are treated with $50 \mathrm{nM}$ Taxol. In contrast, the percent of live H460-R cells is $77.5 \%$ and $64 \%$ when treated with $10 \mathrm{nM}$ and $50 \mathrm{nM}$ Taxol, respectively (fig.3.1B). In conclusion, we confirmed that H460-R cells are resistant to Taxol treatment.
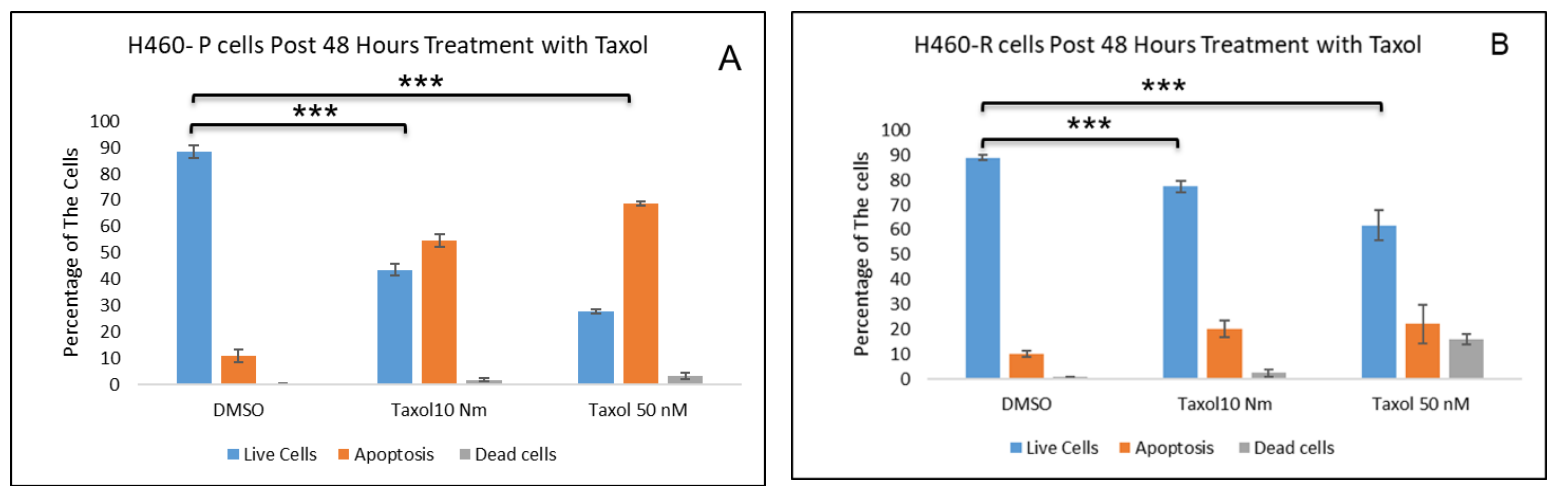

Figure 3. 1. H460-R cells are Taxol-resistant. H460-P (A) and H460-R (B) cells were treated either with DMSO or Taxol with the indicated doses. After 48 hours, the cells were stained with Annexin V and PI and the percent of live/dead cells was determined by flow cytometry. Error bars represent standard deviation from three biological replicates. A mixed effect model was used to assess the difference between two conditions, using $R$ package Ime4. The p-value was calculated based on asymptotic Z-distribution

\subsection{Validation of the ZNF71 Antibodies by Overexpression of the Protein}

We wished to validate the ZNF71 antibodies purchased from Sigma and GeneTex used in our experiments since there are no published data on their specificity. First, the specificity of the antibodies was examined by knocking down ZNF71; this process compares the antibody bands of 
knocked down cells to the wild type cell signal. We were unable to detect the endogenous ZNF71; for this reason, we next elected to overexpress ZNF71 to determine whether the antibodies recognize ZNF71 protein. HEK-293 cells were transfected with ZNF71 expression tagged with V5 as well as with plasmids expressing GFP, ZNF175, and ZNF263 controls. The overexpression was validated with qRT-PCR assay. As shown in figure 3.2 (A), the ZNF71 mRNA expression is about 200 times higher in the ZNF71-overexpressing cells than in the control cells. Both ZNF71 antibodies detected a protein band corresponding to ZNF71 detected and matched the signal of anti-V5 antibody (Fig.3.2.B) while no signals were detected in any control group. We conclude that while both ZNF71 antibodies purchased from Sigma and GeneTex cannot detect endogenous levels of ZNF71 protein, they can detect the higher level of ZNF71 by overexpression.
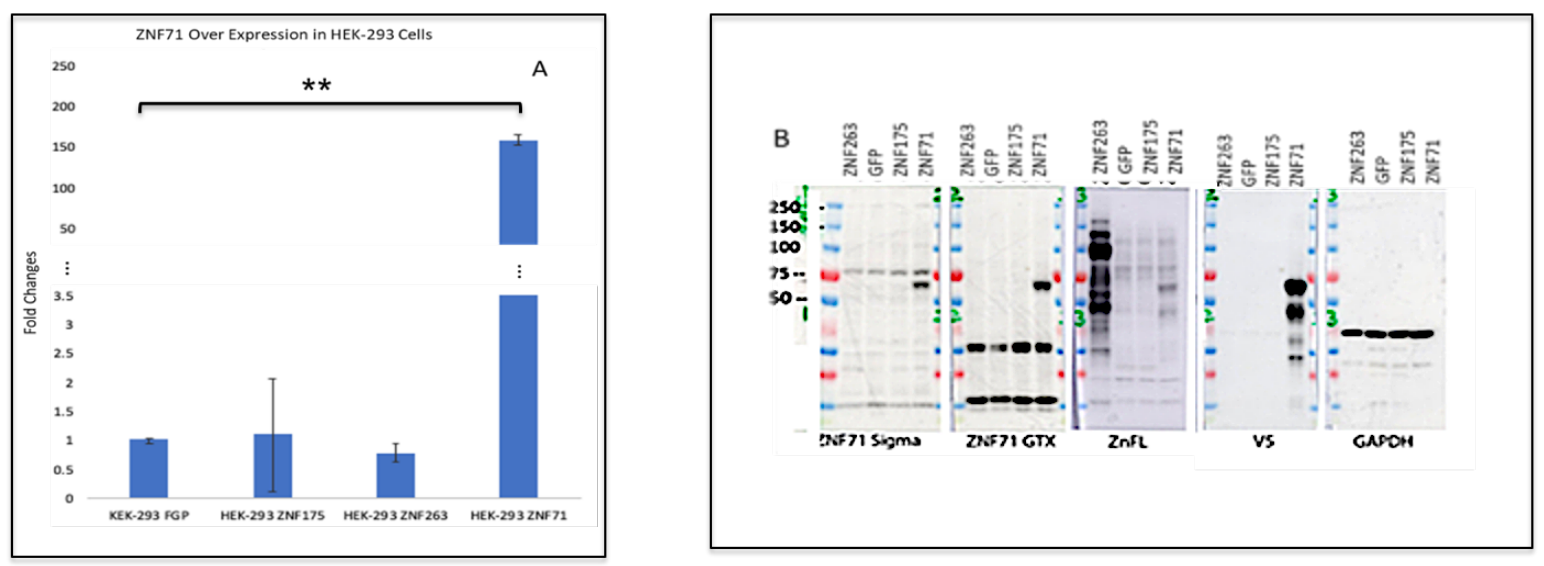

Figure 3. 2: ZNF71 antibodies validation. A qRT-PCR assay was performed to measure ZNF71 mRNA expression. Error bars represent standard deviation from three technical replicates. B. ZNF71 protein detection by two antibodies from Sigma and GeneTex. The band was confirmed by the V5 antibody that labeled the plasmid. GAPDH is a loading control.

\subsection{ZNF71 mRNA Levels are Higher in H460-P than in H460-R}

Once we confirmed the resistance of H460-R cells to Taxol, our next experiment was to study whether there is a difference in the ZNF71 mRNA level between the resistant and parental cells. The two cell lines were plated in six-well plates in six biological replicates. For DMSO and Taxol treatment, H460-P and H460-R cells were plated in three biological replicates. RNA was collected from each replicate for qRT-PCR, (see Methodology) as shown in figure 3.2(A). The ZNF71 mRNA level is higher in the H460-P cells, even when the cells were treated with Taxol, (Fig.3.2.B). It was also noted that the ZNF71 mRNA levels increase with Taxol treatment. 

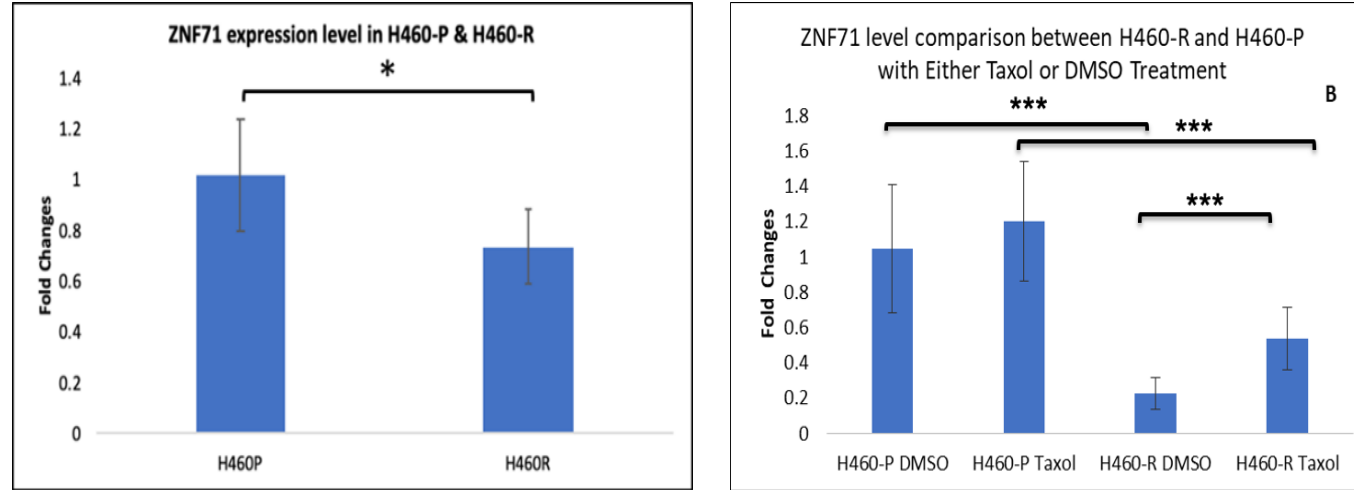

Figure 3. 3. A. Comparison of the ZNF71 mRNA levels in resistant cells, H460-R, and parental cells, H460-P. Six biological replicates for H460-P and H460-R were plated. RNA was isolated and subjected to qRT-PCR. B. Both H460-P and H460-R cells were treated with either Taxol or DMSO, then the level of ZNF71 mRNA was quantified by qRT-PCR. Error bars represent three biological replicates. ZNF71 mRNA levels are higher in

H460-P; Taxol treatment leads to slight increase in the level of ZNF71 mRNA. A mixed effect model was used to assess the difference between two conditions, using R package Ime4. The p-value was calculated based on asymptotic Z-distribution

\subsection{H460-R Cells Response to Taxol Treatment Slightly Affected by Changing the Levels of ZNF71}

To study whether we observe a direct impact on the cells' response to Taxol chemotherapy, ZNF71 was knocked down using ZNF71 siRNA. After 72 hours, the cells were treated with either vehicle control DMSO, $10 \mathrm{nM}$ or $50 \mathrm{nM}$ Taxol. After further 48 hours treatment with Taxol, the cells were stained with Annexin V and PI to measure the apoptotic cell death. As shown in figure 3.3, the cells response to chemotherapy was slightly affected by ZNF71 knockdown, cell survival increased and cell death decrease after ZNF71 gene knockdown with respect to the treatment doses. For this experiment, we planned to redesign it in a way that we can collect RNA at the time of transfection instead of after exposure to Taxol to measure the knockdown effectiveness, and correlate it to the cellular response to Taxol. Unfortunately, as many other experiments- explained later in Restrictions and Limitations section- this work was stopped because of COVID-19 social distancing regulations. 


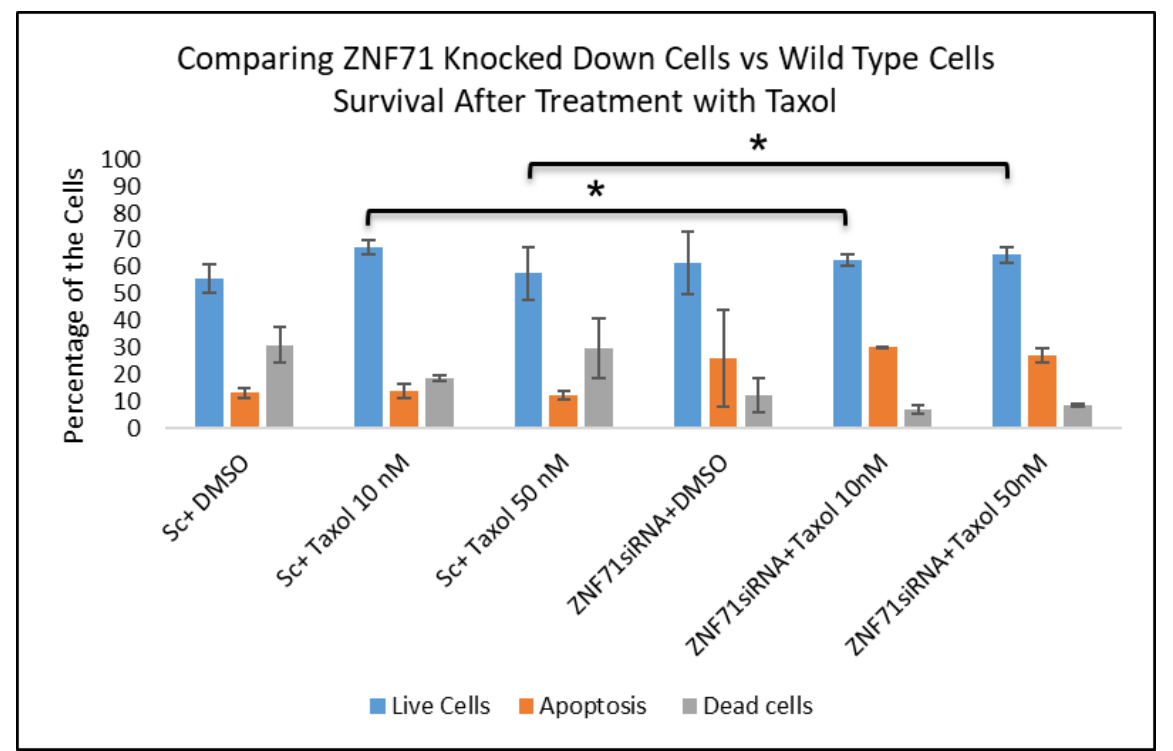

Figure 3. 4 Effect of ZNF71 gene knockdown on H460-R cells response to Taxol. H460-R cells were transfected with ZNF71 or scrambled control siRNAs and after 72 hours the cells were treated with either DMSO, 10 nM, or 50 nM Taxol for 48 hours. Cells were collected and stained with Annexin V and PI and percent of live, apoptosis, and dead cells were determined by follow cytometry. The ZNF71 knockdown had a mild effect on the Taxol response of the cells. A mixed effect model was used to assess the difference between two conditions, using $R$ package Ime4. The p-value was calculated based on asymptotic Z-distribution

\subsection{ZNF71 is Expressed in Different Amounts in Different non-Small Cell Lung Cancer Cells}

We performed an experiment to determine the level of expression of ZNF71 in various NSCLC cell lines. Multiple NSCLC were plated in six wells plates at a confluence of $80 \%$, then mRNA and the protein were purified from each cell line. We quantified the mRNA using qRTPCR, using NHBE cells as a control for the cancer group. We also performed a Western blot to determine the ZNF71 protein level using overexpressed ZNF71 in HEK-293 cells as a control. The endogenous level of the protein was not detectable in any of the cell lines tested, with only nonspecific bands appearing on the membrane. Next, we classified the cell lines as either mesenchymal or epithelial according to the signals detected by the Western blot using known mesenchymal and epithelial markers, like ZEB1 for mesenchymal and E-Cad for epithelial cells. As shown in figure 3.5, the cells are divided into three groups, 1) epithelial cell lines, NHBE, H358, H441, H513, and H820; 2) mesenchymal cell lines, H23, H460, and H1299, and, 3) mostly mesenchymal cell lines, A549 and H1395. Next we compared the level of ZNF71 mRNA expression between these cell groups. The level of ZNF71 was relatively higher among epithelial cells, lower among the mesenchymal group, and intermediate in the mostly-mesenchymal cells group. There was a plan to repeat this experiment with plating every cell line in four biological replicates, three replicates 
to be used to collect the RNA, and the fourth for protein. Unfortunately, COVID-19 social distancing regulations stopped us from finishing this experiment.
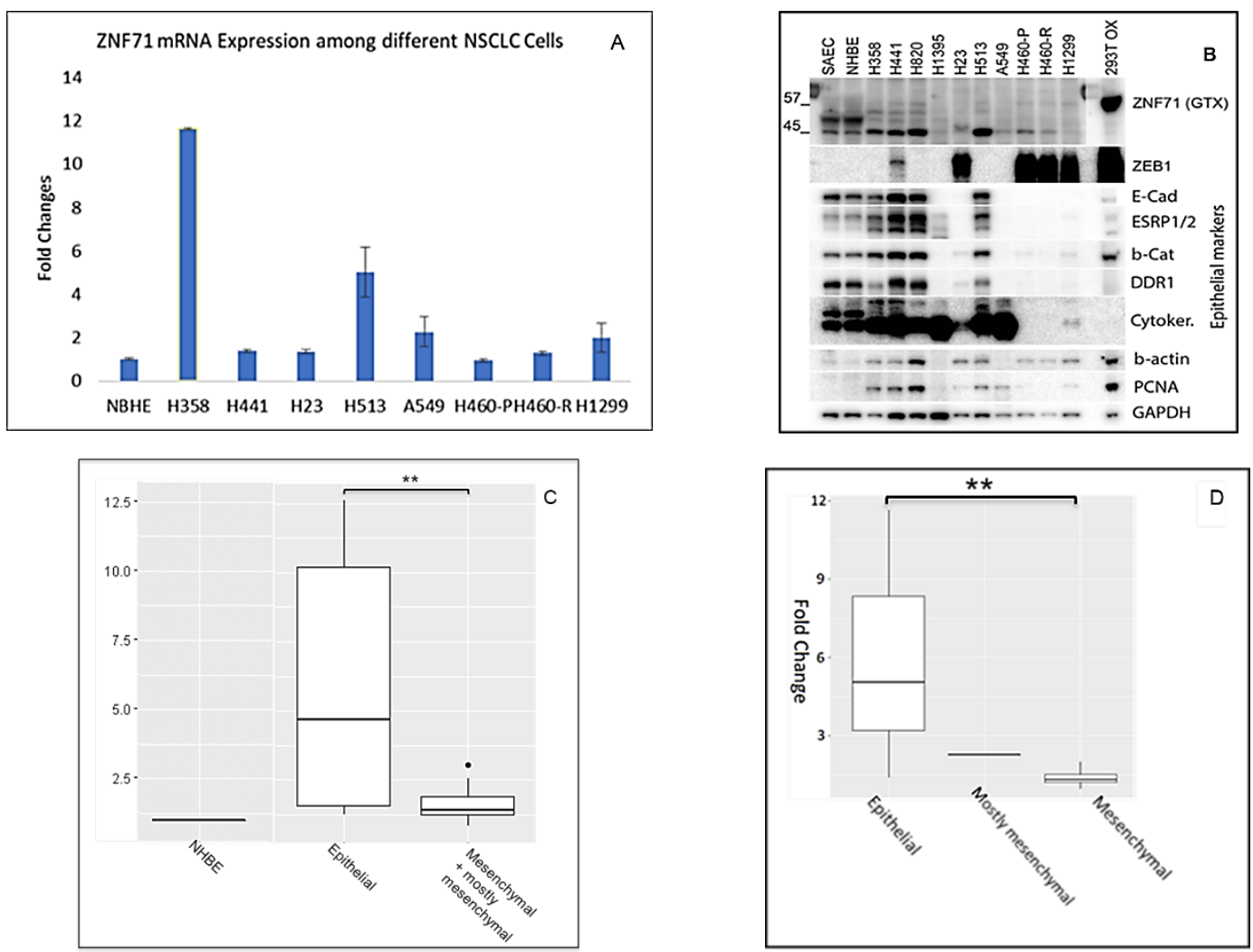

Figure 3. 5. ZNF71 level is different in epithelial and mesenchymal cell lines. A, ZNF71 mRNA levels among different NSCLCs. B, Anti-ZNF71 antibodies detect overexpressed but not the endogenous protein level in NSCLC cell lines. Classification of NSCLC cells according to epithelial and mesenchymal markers, some cell lines express E-Cadherin (epithelial), other express ZEB1 (mesenchymal), while mostly mesenchymal neither express E-Cad nor ZEB1. C, ZNF71 mRNA levels are higher in epithelial cell lines either when compared to

combined mesenchymal and mostly mesenchymal or $D$, when compared to mesenchymal and mostly mesenchymal separately.

\subsection{The KRAB-ZNF71 Isoform is Expressed Mainly in Mesenchymal NSCLC Cells While the KRAB-Less Isoform Is Expressed in the all Cell Lines, Including Primary Non- Cancerous Cells.}

Because of the importance of KRAB-ZNF and its relation to some tumor recurrence and metastasis, we decided to determine the relative ratio of the two isoforms in different cell lines. We designed three sets of primers that amplify either both isoforms, or the KRAB isoform and KRAB-less isoform separately. First, we measured in which cell line each isoform was expressed and what was the relationship between KRAB-ZNF71 isoform and mesenchymal-epithelial cell 
lines. As shown in figure 3.6, mostly mesenchymal NSCLC cells express both the KRABcontaining and KRAB-less isoforms, while the epithelial cells express only the KRAB-less isoform. Also, in most of the cell lines, the KRAB-less ZNF71 level was higher than the KRABZNF71 except for H460-R, which was derived from H460-P. In this cell line, the level of the $\mathrm{KRAB}$ isoform is much higher than the level of the KRAB-Less isoform. This could be related to the cells resistance to Taxol and it could be induced by Taxol when the cells were treated with it at the time of developing resistance to Taxol. The next experiment planned was to knock down and overexpress the KRAB-ZNF71 isoform in one cell line per mesenchymal, epithelial, and mostly mesenchymal cell groups, then study the effect of this gene manipulation on the epithelial and mesenchymal markers expression in these cells. Moreover, studying the response of the cell to Taxol after transfection was also planned. This plan was prevented by the rule of COVID1social distancing.

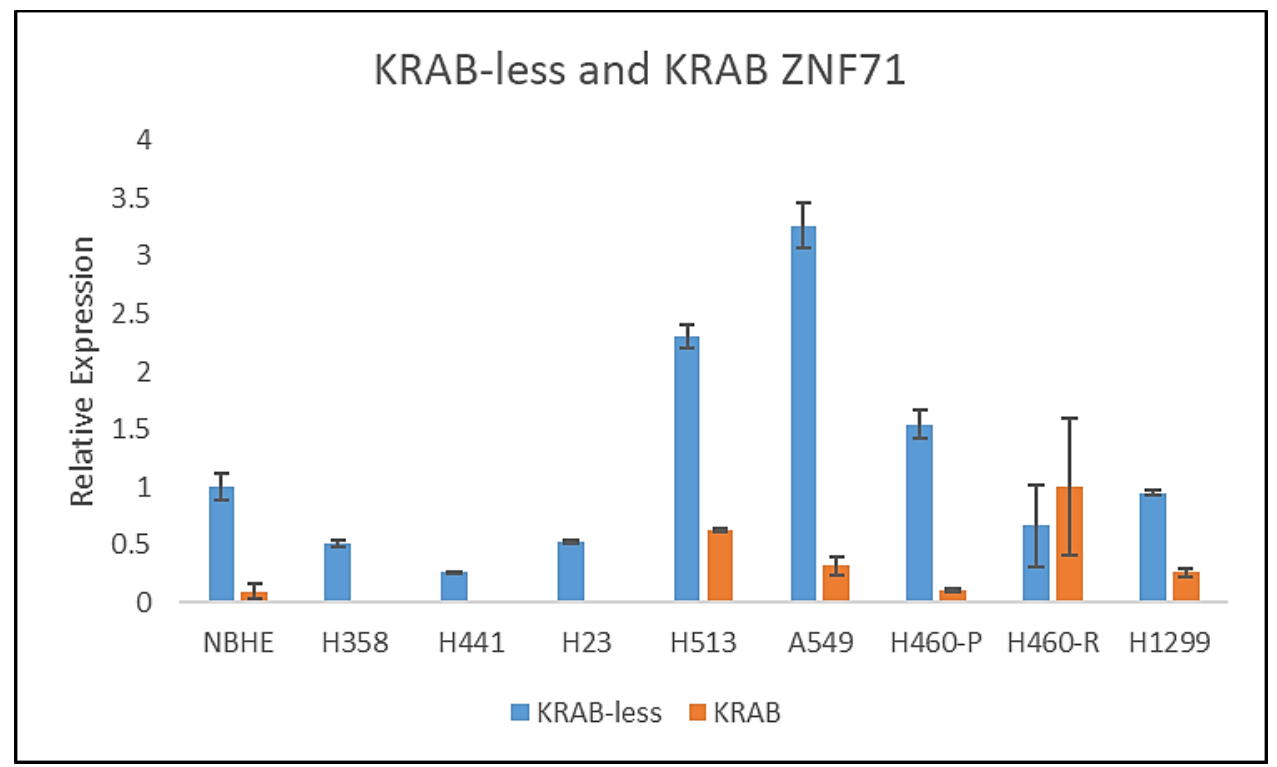

Figure 3. 6 Relative expression ZNF71 mRNA isoform among different NSCLC cell lines. $q R T$-PCR with primers detecting either the KRAB-less or KRAB-containing ZNF71 isoforms. KRAB-ZNF71 is detected predominantly in mesenchymal cells. Increase the power of the experiment by growing every cell line in three biological replicates to get the statistical significance. Error bars represent the technical replicates. 


\subsection{Conclusions}

\section{CHAPTER 4}

ZNF71 is one of the KRAB zinc finger protein family which could be associated with tumor prognosis and patients outcomes. It also reported in our previous study that patients with higher protein expression have better survival and chemo-sensitivity to the chemotherapy. In this project, we studied the molecular functions of ZNF71 in NSCLC. ZNF71 mRNA was expressed slightly higher in H460-P cells than H460-R cells with concordant to our previous study, which indicates the higher level of ZNF71 the better patients' survival and better respond to chemotherapy. Furthermore, we interested in the finding that total ZNF71 mRNA is higher in epithelial than in mesenchymal NSCLC cell lines, and we planned further molecular and bioinformatics investigations. KRAB containing ZNF71 was expressed predominantly in mesenchymal NSCLCs, while KRAB-less ZNF71 expressed in both mesenchymal and epithelial NSCLC. KRAB-less ZNF71 mRNA level is higher than KRAB-ZNF71 in all studied cell types except in H460-R cells which could be related to Taxol resistance. Because of the regulation of COVID-19 social distancing, many experiments were recommended to be resumed at later date to study the gene further.

\subsection{Future Plans}

1. Clone KRAB containing plasmid for over expression experiment.

2. Design KRAB-ZNF71 siRNA specific to the exon coding for KRAB domain for gene knock down experiments.

3. Repeat the redesigned experiment of the ZNF71 manipulated cells. In a future experiment, we will use the KRAB-ZNF71 siRNA, and the cells will be plated in 6-well plates, with every group in three biological replicates. RNA will be isolated 48-hours post transfection. Further, transfected cells will be treated with $10 \mathrm{nM}$ Taxol for 48 hours. The cells will then be stained with Annexin V and PI to calculate the percentage of the live, apoptotic, and dead cells.

4. Overexpression of KRAB containing ZNF71, collect RNA and treat the cells with Taxol 48 hours post transfection. After 48 hours treat the cells with Taxol and collect the cells for Annexin V and PI staining to measure live, apoptotic, and dead cells.

5. Repeat the experiment for ZNF71 level and KRAB-ZNF71 in three biological replicates. 
What I have learned during my study in the program?

- Cell culture.

- Cellular transfection and gene knockdown

- Protein overexpression

- RNA isolation using both Trizol and centrifugation techniques

- cDNA synthesis

- qRT-PCR assay

- MTT assay

- Western blot assay

- Annexin V and PI staining and flow cytometry

- I learned how to read scientific papers

- Scientific writing

\section{Classes taken during my course work}

- Foundations 1 and 2

- Scientific integrity

- Cellular methods

- Molecular Genetic

- Cancer cell Biology

- Scientific Writing

- Cancer Pharmacol/Theraputics

- Biomedical/ Oncogenic signaling

- Journal Clubs 


\section{References}

1. Klug A. The discovery of zinc fingers and their development for practical applications in gene regulation and genome manipulation. Q Rev Biophys. 2010. doi: $10.1017 /$ S0033583510000089

2. Laity JH, Lee BM, Wright PE. Zinc finger proteins: New insights into structural and functional diversity. Curr Opin Struct Biol. 2001. doi:10.1016/S0959-440X(00)00167-6

3. Cassandri M, Smirnov A, Novelli F, et al. Zinc-finger proteins in health and disease. Cell Death Discov. 2017. doi:10.1038/cddiscovery.2017.71

4. Chew A. The role of KRAB-ZFPs in transposable element repression and mammalian evolution. Q Rev Biol. 2017;82(3):251-255. doi:10.1086/519967

5. Peng H, Begg GE, Harper SL, Friedman JR, Speicher DW, Rauscher FJ. Biochemical analysis of the Kruppel-associated box (KRAB) transcriptional repression domain: Spectral, kinetic, and stoichiometric properties of the KRAB·KAP-1 complex. J Biol Chem. 2000;275(24):18000-18010. doi:10.1074/jbc.M001499200

6. Ecco G, Imbeault M, Trono D. KRAB zinc finger proteins. Dev. 2017. doi:10.1242/dev.132605

7. Ho J, Kong J, Choong L, et al. Novel Breast Cancer Metastasis-Associated Proteins research articles. 2009:583-594. doi:10.1021/pr8007368

8. Addison JB, Koontz C, Fugett JH, et al. KAP1 promotes proliferation and metastatic progression of breast cancer cells. 2015;75(2):344-355. doi:10.1158/0008-5472.CAN-141561.KAP1

9. Zhang $\mathrm{X}$, Jiang $\mathrm{G}, \mathrm{Wu}$ J, Zhou $\mathrm{H}$. Zinc finger protein 668 suppresses non-small cell lung cancer invasion and migration by downregulating Snail and upregulating E-cadherin and zonula occludens-1. 2018:3806-3813. doi:10.3892/ol.2018.7802

10. Mataki C, Murakami T, Umetani HM, Youichiro T, Ishii M, Kodama S. Finger Protein Induced mRNA in Human Necrosis Umbilical Factor a Vein Endothelial is Profoundly by Tumor Hiroyuki Takao Masami scription ( Ambion ) was performed with biotinylated UTP and CTP (Enzo Diagnostics ), resulting in 40- to 80-fold linear amplif. $J$ Atheroscler Thromb. 2000;7.

11. Lan N, Dowlati A, Raese RA, et al. EBioMedicine A Predictive 7-Gene Assay and Prognostic Protein Biomarkers for Non-small Cell Lung Cancer. EBioMedicine. 2018;32:102-110. doi:10.1016/j.ebiom.2018.05.025

12. Pearce MC, Gamble JT, Kopparapu PR, et al. Induction of apoptosis and suppression of tumor growth by Nur77-derived Bcl-2 converting peptide in chemoresistant lung cancer cells. 2018;9(40):26072-26085.

13. Dakhlallah DA, Wisler J, Gencheva M, et al. Circulating extracellular vesicle content reveals de novo DNA methyltransferase expression as a molecular method to predict septic shock. J Extracell Vesicles. 2019;8(1). doi:10.1080/20013078.2019.1669881 